\title{
Isavuconazole: Mechanism of Action, Clinical Efficacy, and Resistance
}

\author{
Misti Ellsworth ${ }^{1, *}$ and Luis Ostrosky-Zeichner ${ }^{2}$ \\ 1 Division of Pediatric Infectious Diseases, UT Health McGovern Medical School, Houston, TX 77030, USA \\ 2 Division of Infectious Diseases, UT Health McGovern Medical School, Houston, TX 77030, USA; \\ Luis.Ostrosky-Zeichner@uth.tmc.edu \\ * Correspondence: misti.ellsworth@uth.tmc.edu; Tel.: +1-713-500-5312
}

Received: 13 October 2020; Accepted: 21 November 2020; Published: 29 November 2020

\begin{abstract}
Increasing incidence of invasive fungal infections combined with a growing population of immunocompromised hosts has created a rising need for antifungal agents. Isavuconazole, a second-generation broad-spectrum triazole with activity against yeasts, dimorphic fungi, and molds, has a favorable safety profile and predictable pharmacokinetics. Patients typically tolerate isavuconazole well with fewer drug-drug interactions. Clinical trials have found it to be noninferior to voriconazole for invasive aspergillosis, an alternative therapy for salvage treatment of mucormycosis, and suitable for stepdown therapy with invasive candidiasis. Cross-resistance with other triazoles is common. More studies are needed to determine the role of isavuconazole in anti-mold prophylaxis in high-risk patients.
\end{abstract}

Keywords: isavuconazole; triazole; Aspergillus; Candida; Mucorales; invasive fungal disease

\section{Introduction}

The incidence of invasive fungal infections (IFI) has been increasing as advances in medical treatments for cancer and other chronic health conditions create an ever-growing population of immunocompromised hosts. The mortality rate of these infections is estimated to exceed $50 \%$ [1]. The high mortality rate in combination with limited treatment options leads to over 1.5 million deaths/year worldwide [2]. Four classes of antifungal drugs comprise the arsenal to combat IFIs: azoles, echinocandins, polyenes, and flucytosine. Within the azole class, the systemic use triazoles are some of the most frequently used antifungal drugs due to improved tolerability and more favorable side effect profile when compared to polyenes [3]. One of the newest triazoles, isavuconazole, was approved by the FDA in 2015 for the treatment of invasive aspergillosis and mucormycosis. Its use has since been expanded to treat a wide array of fungal infections and as IFI prophylaxis. The aim of this review is to review the mechanism of action of isavuconazole, summarize the treatment data available, and explore mechanisms of resistance.

\section{Mechanism of Action}

Isavuconzaole inhibits cytochrome P450-dependent lanosterol $14 \alpha$-demethylase, which is essential for the synthesis of ergosterol, a component of the fungal membrane. This disruption leads to alterations in the structure and function of the fungal membrane leading to cell death [4]. The isavuconazole structure includes a side arm that orients the molecule to engage the triazole ring to the binding pocket of the fungal CYP51 protein (Figure 1). This confers broader antifungal activity in comparison to other azoles [5]. 


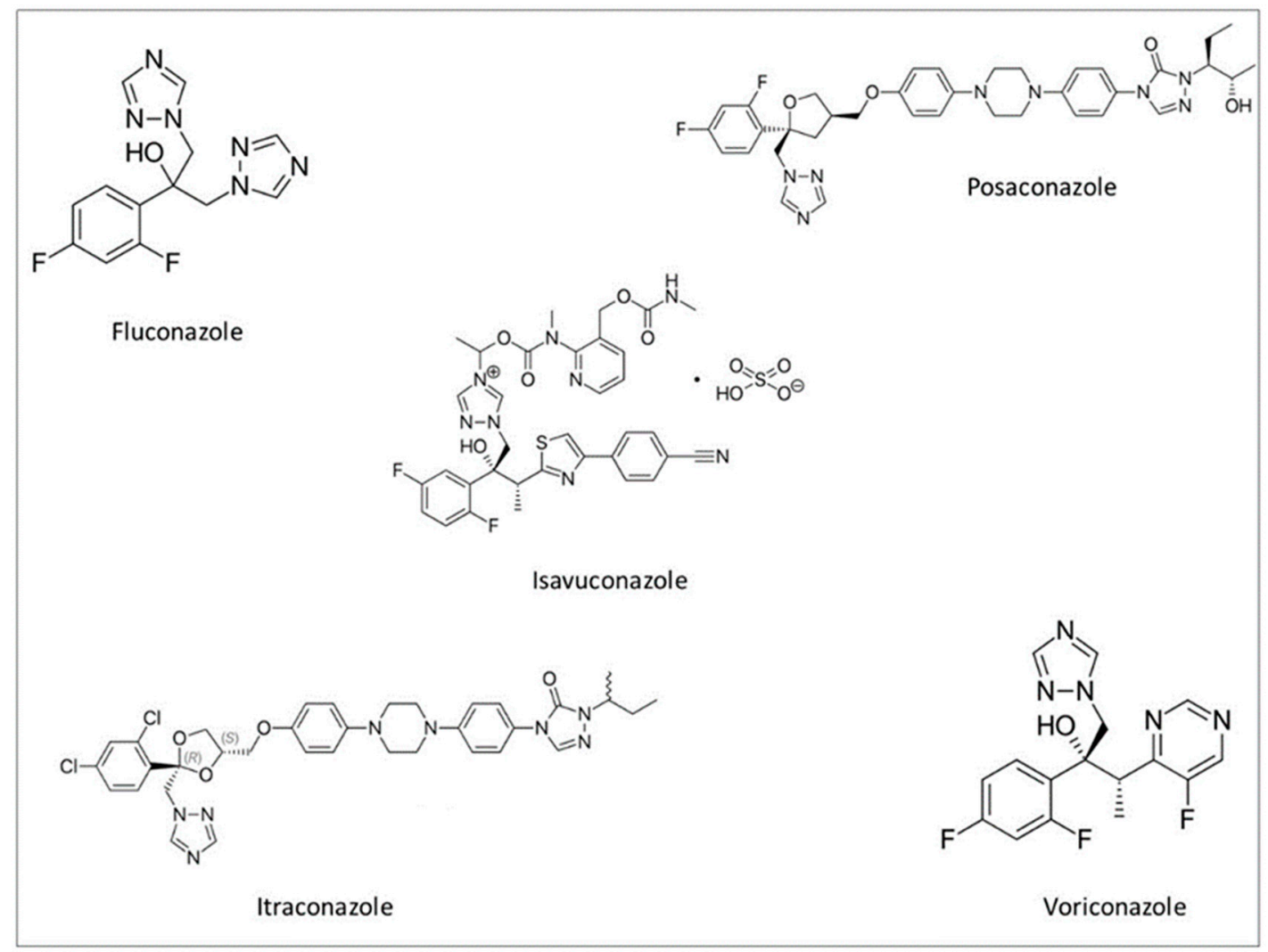

Figure 1. Chemical structure of fluconazole, posaconazole, isavuconazole, itraconazole, and voriconazole.

\section{Pharmacokinetics and Pharmacodynamics}

Isavuconazole is the active component of isavuconazonium sulfate, a triazole precursor that is water-soluble and can be given orally or intravenously. The drug has a large volume of distribution with the majority (approx. 98\%) being bound to protein [4]. Bioavailability is high, approaching $98 \%$, and the same dose is used for oral and intravenous administration. Unlike other triazoles, isavuconazole absorption is not impacted by food intake, gastric acid-suppressing medications, or the presence of mucositis [6,7]. Previous studies have proven that isavuconazole administered in an IV or oral formulation follows dose-dependent pharmacokinetics with minimal variability among healthy subjects with the maximum plasma concentration occurring one and two to three hours after administration, respectively [8,9]. Isavuconazole is proposed to be widely distributed to the majority of tissues, including brain, liver, lung, and bone. Clinical data supporting brain penetration in humans is limited to case reports showing successful treatment of fungal CNS infections with isavuconazole [10]. Tissue distribution has been studied in rat models for both single and daily doses of isavuconazole, with findings of rapid tissue penetration [11]. The VITAL and SECURE trials showed similar pharmacokinetics with minimal intra-subject variability and narrow distributions of trough levels in patients with proven IFIs $[12,13]$. Similar results were found in trough levels of patients with renal failure [14]. Subsequent studies have found disparities in drug levels in patients on renal replacement therapy and extracorporeal membrane oxygenation (ECMO), and Andres et al. found therapeutic drug monitoring (TDM) may be warranted in patients who are obese, $<18$ years of age, or who have moderate hepatic failure [15-17]. Currently, neither the European Conference on Infections in Leukemia (ECIL-6) guidelines nor the Infectious Disease Society of America Guidelines for Aspergillosis recommend routine TDM for isavuconazole [18,19]. 


\section{In Vitro Activity}

In vitro data support broad activity for isavuconazole against yeast, molds, and dimorphic fungi. Isavuconzole is active against most Candida species, including C. krusei and C. glabrata [20]. In a study that compared voriconazole and isavuconazole minimum inhibitory concentrations (MIC) of 1677 Candida isolates found the distributions were comparable [21]. Seifert et al. evaluated 296 blood stream Candida isolates for in vitro activity of amphotericin B, flucytosine, fluconazole, itraconazole, voriconazole, and isavuconazole. Isavuconazole was found to have lower MICs than fluconazole and similar activity to amphotericin B, itraconazole, and voriconazole. The minimum inhibitory concentration (MIC) values for all major Candida species, corresponding to $50 \%$ and $90 \%$ growth inhibition, were as follows: MIC50 $<0.5 \mathrm{mg} / \mathrm{L}$ and MIC90 $<2.0 \mathrm{mg} / \mathrm{L}$, respectively [22]. In addition to Candida, in vitro activity is also present for Cryptococcus neoformans and Cryptococcus gattii with MICs reported from $<0.008$ to $0.5 \mathrm{mg} / \mathrm{L}$ [23]. Isavuconazole activity against $C$. auris is variable, and empiric therapy should be initiated with an echinocandin until susceptibility results are available [24].

Isavuconazole plays an important role in the treatment of invasive aspergillosis. In a study evaluating 702 strains of Aspergillus, isavuconazole showed an MIC90 of $1 \mu \mathrm{g} / \mathrm{mL}$. Isavuconazole also showed activity against $A$. terreus, a known amphotericin B resistant species [20]. In a report that evaluated isavucoazole's in vitro activity against typically azole-resistant fungal pathogens including A. lentulus found isavuconazole was active despite voriconazole and itraconazole resistance [25].

A total of 72 clinical isolates of Mucorales were evaluated for susceptibility to amphotericin B, posaconazole, voriconazole, and isavuconazole. All isolates were susceptible to amphotericin, resistant to voriconazole, and potentially susceptible to posaconazole and isavuconazole. Of note, more isolates were found to be potentially susceptible to isavuconazole when compared to posaconazole [26].

Small studies have also shown potential activity against Scedosporium and Fusarium species; however, more in vitro data are needed before strong conclusions can be made [20].

\section{Drug-Drug Interactions and Adverse Events}

Overall, isavuconazole is well tolerated with a favorable side effect profile when compared to other agents in the azole class. The most commonly reported side effects include nausea, vomiting, and diarrhea $[9,21]$. Symptoms are generally not severe enough to require discontinuation of therapy. Hepatotoxicity can occur with isavuconazole and liver enzymes should be monitored while on therapy. Overall, this occurs to a lesser extent than with other commonly used triazoles. In the SECURE trial, there was a lower frequency of drug-related hepatotoxicity in the isavuconazole group versus the voriconazole group ( $2 \%$ vs. $10 \%$ [27]).

In contrast to other triazoles that cause prolongation of the time duration between the onset of the QRS complex and the end of the T wave, or QTc segment, isavuconazole can lead to dose-dependent QTc shortening [28]. A paper evaluating changes in the QTc interval in 26 adult patients found that 24 patients showed a mean interval decrease of $7.4 \pm 5.8 \%$ [29]. With the exception of individuals with familial shortened QTc syndrome, in whom isavuconazole should be avoided, the clinical significance of QTc interval shortening remains unclear [30].

Isavuconazole is a substrate for CYP3A4, and coadministration of other medications that inhibit or induce this enzyme should be avoided. Inhibitors that can lead to increased isavuconazole levels include ketoconazole and high-dose ritonavir, and the inducers that can lead to reduced levels of isavuconazole include long-acting barbiturates, rifampin, and carbamazepine [30]. Isavuconazole is a moderate inhibitor of CYP3A4 and can lead to elevated levels of drugs such as sirolimus, tacrolimus, cyclosporine, colchine, and digoxin due to decreased metabolism. Van Kieu et al. evaluated tacrolimus/sirolimus levels in hematopoietic stem cell transplants receiving isavuconazole and found a moderate increase in the concentration/dose ratios. Serum levels of these drugs should be monitored if given concomitantly with isavuconazole [31]. 


\section{Clinical Efficacy}

\subsection{Invasive Aspergillosis}

Invasive aspergillosis (IA) accounts for the majority of IFIs in immunocompromised patients and results in significant mortality and morbidity. A study in hematopoietic stem cell transplant (HSCT) patients found the overall one-year survival rate to be $25.4 \%$ in patients with IA infections [32]. Treatment options for IA are limited, and the standard drugs used in treatment have significant toxicities and drug interactions. To confirm the in vitro data for isavuconazole against Aspergillus species, the SECURE trial was designed to compare treatment outcomes for isavuconazole versus voriconazole in patients with suspected invasive mold disease [27]. This was a Phase 3 randomized double-blind non-inferiority trial. The primary endpoint was all-cause mortality at day 42 of treatment in patients who received at least one dose of the study drug using a 10\% non-inferiority margin. Secondary endpoints included all-cause mortality at day 84 , overall clinical, mycological, and radiological response at day 42,84 , and the end of treatment. Safety and tolerability of both drugs was also studied. A total of 527 patients were randomized to receive either voriconazole $(n=264)$ or isavuconazole $(n=263)$ at the following doses: voriconazole $6 \mathrm{mg} / \mathrm{kg}$ IV twice daily on day one, $4 \mathrm{mg} / \mathrm{kg}$ IV twice daily on day two, followed by either IV treatment at $4 \mathrm{mg} / \mathrm{kg}$ IV twice daily or $200 \mathrm{mg}$ orally twice daily or isavuconazole $200 \mathrm{mg}$ IV three times daily on days one and two followed by $200 \mathrm{mg}$ IV once daily IV or perorally. In each group, 258 patients received at least one dose of the study drug. Probable, proven, or possible invasive mold disease was determined using the definitions from the European Organization for Research and Treatment of Cancer/Invasive Fungal Infections (EORCT) and was confirmed by independent infectious disease experts. In total, $65(13 \%)$ patients were determined to have proven disease, 207 (40\%)—probable disease, 196 (38\%)—possible disease, and 48 (9\%) had no evidence of disease. The most common underlying condition were hematologic malignancies $(84 \%)$, of which $66 \%$ were neutropenia; in $20 \%$ of cases, patients received allogenic hematopoietic stem cell transplants. From these patients, two modified populations were created for further analysis. The modified intention-to-treat (mITT) population was created to include ITT patients with proven or probable invasive mold disease, and a mycological intention-to-treat population (myITT) was a subset of the mITT population with proven or probable IA. When Aspergillus was cultured as the only mold at the baseline, A. fumigatus, A. flavus, A. niger, and A. terreus were the most commonly identified species. Efficacy analysis for treating invasive aspergillosis focused on the mITT population.

The primary endpoint of all-cause mortality at day 42 found that isavuconazole was non-inferior to voriconazole in the ITT population $(18.6 \%$ for the isavuconazole arm and $20.2 \%$ for the voriconazole arm). Overall success, including complete and partial response, at the end of treatment was not different for the two drugs ( $35 \%$ isavuconazole, $3.4 \%$ voriconazole). The results were similar for the mITT and myITT groups for all-cause mortality ( $20 \%$ versus $23 \%$ and $19 \%$ versus $22 \%$, respectively) and treatment response (35\% vs. $36 \%$, respectively). In addition, isavuconazole was associated with significantly less hepatobiliary, eye, and skin adverse events and treatment discontinuation due to adverse events was significantly lower in the isavuconazole arm.

A separate retrospective study found similar results in patients on long-term therapy for chronic pulmonary aspergillosis. Patients who received isavuconazole had significantly less adverse events when compared to patients treated with voriconazole [33].

Using economic modeling, cost-effectiveness studies have since been done in the US, the UK, and Sweden. All studies found that isavuconazole was cost-saving when compared to voriconazole. These cost savings included the cost of the drug, the cost of adverse events, and hospital readmissions [34-36].

Current guidelines from ECIL-6 and the European Society of Clinical Microbiology and Infectious Diseases/European Confederation of Medical Mycology recommend either voriconazole or isavuconazole as the first-line treatment for IA in patients with hematological malignancies $[19,37]$. Infectious Disease Society of America (IDSA) guidelines for the treatment of IA still recommend voriconazole as the first-line treatment [18]. 


\subsection{Mucormycosis}

Mucormycosis is a rare disease occurring primarily in patients who are immunocompromised. Prompt diagnosis and treatment is necessary due to the significantly high mortality rate of up to $90 \%$ associated with these infections. Treatment guidelines center on antifungal treatment and surgical debridement [38]. Anti-fungal agents used in treatment include amphotericin B and posaconazole. Both agents are associated with significant toxicity and adverse events. In 2015, the FDA approved the use of isavuconazole for invasive mucormycosis based on the results of the VITAL study. The VITAL study was an open-label non-comparative study of isavuconazole in adult patients with IA and renal impairment or in patients with invasive fungal disease caused by other rare fungi [39]. Within this study, 37 patients had proven $(86 \%)$ or probable $(14 \%)$ invasive mucormycosis as defined by the European Organization for Research and Treatment of Cancer/Mycosis Study Group definition. Patients with mucormycosis were eligible for analysis if isavuconazole was the primary treatment (defined as 4 days or less) or if they were refractory or intolerant to other antifungals. Of the 37, 21 patients had not received anti-fungal therapy, 11 had refractory disease, and 5 were intolerant of previous therapy. The most common underlying condition was hematologic malignancy (59\%) with $35 \%$ having received HSCT and $27 \%$ being neutropenic. Disease involvement was pulmonary in $59 \%$ with additional site involvement in $1 / 2$. Patients received an oral or IV loading regimen of isavuconazole $200 \mathrm{mg}$ every 8 hours for six doses followed by $200 \mathrm{mg}$ daily. Clinical efficacy of isavuconazole was also assessed using matched case-control analysis from the FungiScope: Global Emerging Fungal Infection Registry, a global database of rare invasive fungal diseases. This was in line with the FDA guidance for comparators in studies of rare diseases. In this analysis, patients with the isavuconazole primary treatment were matched up with three FungiScope patients who had received primary amphotericin B-based treatment for proven or probable mucormycosis [39].

The primary VITAL study endpoint was the overall clinical response at day 42 . Secondary endpoints included assessments of overall, clinical, radiological, and mycological responses at day 42 , day 84 , and the end of treatment and all-cause mortality at days 42 and 84 . Mortality at day 42 was $38 \%$. When mortality was compared to patients with refractory disease or previous intolerance of prior therapy versus patients who were treated for primary mucormycosis, mortality was higher in the refractory/intolerance group ( $43.7 \%$ vs. $33.3 \%)$. The overall response rate at day 42 was $31.4 \%$. End of treatment partial and compete response was $32 \%$ for primary treatment and $36 \%$ for refractory treatment. These rates were similar to those reported for amphotericin B.

The matched case-control analysis compared 21 patients from the VITAL trial who had primary treatment with isavuconazole to 33 patients treated with amphotericin from the FungiScope Registry. The all-cause mortality at day 42 and survival through day 84 did not differ between the two groups. Similar to the SECURE study, the VITAL study found isavuconazole to be well tolerated with seven patients able to extend therapy beyond 6 months [40].

Several other case reports have subsequently shown isavuconazole to be effective as the salvage therapy for mucormycosis [41-43].

\subsection{Candida}

Incidence of invasive candidiasis (IC) is increasing within the United States. Hospital inpatient IC is estimated to be $90 / 100,000$ hospitalizations with mortality in patients with IC estimated to be around $40 \%$ [44,45]. Echinocandins are often used as the first-line therapy, but are limited to only IV administration. The ACTIVE trial compared IV isavuconazole to IV caspofungin followed by oral isavuconazole or voriconazole, respectively, in a Phase 3 randomized double-blind clinical trial for the primary treatment of patients with candidemia or invasive candidiasis [46]. Treatment groups received caspofungin $750 \mathrm{mg}$ IV on day 1 followed by $50 \mathrm{mg}$ IV daily or $200 \mathrm{mg}$ of isavuconazole IV q8h on days 1-2 followed by $200 \mathrm{mg}$ once daily. Both groups received IV therapy until day 10. After day ten, if neutropenia was not present, the patient was switched from IV to oral therapy at the clinician's discretion. Doses of oral medications were as follows: voriconazole $400 \mathrm{mg}$ twice daily on 
day 1 followed by $200 \mathrm{mg}$ twice daily on all subsequent treatment days or $200 \mathrm{mg}$ of oral isavuconazole once daily. Treatment continued for a minimum of 14 days after the last positive blood culture and could be extended up to 56 days. Catheter removal was recommended for all patients with candidemia. Patients were followed for 6 weeks, and clinical and laboratory assessments were performed at the baseline, days 7, 14, 28, 42, and 56, the end of IV therapy (EOIVT), and the end of therapy (EOT). All patients who received at least one dose of the study drug were included in the intention-to-treat (ITT, $n=440$ ) population. Patients in the ITT population with invasive candidiasis or candidemia at the baseline were included in a modified ITT $(\mathrm{mITT}, n=400)$ group. The primary efficacy endpoint was the overall response to therapy at the end of IV treatment (EOIVT). A successful response was defined as mycological eradication and clinical cure or improvement without the use of alternative antifungal therapy within 48 hours of the last dose of IV therapy. The secondary endpoint was the overall response to therapy at 2 weeks after the end of therapy (EOT). The mITT population was used to assess the primary and secondary endpoints. The mITT population consisted of 199 patients in the isavuconazole group and 201 in the caspofungin group. The most common Candida species were non-albicans. The most common species in both treatment arms were $C$. albicans, $C$. tropicalis, C. parapsilosis, and C. glabrata.

For the primary endpoint of the overall response at the EOIVT, a successful outcome occurred in $60.3 \%$ cases in the isavuconazole arm and $71.1 \%$ cases in the caspofungin arm. This did not demonstrate noninferiority of isavuconazole in comparison to caspofungin. The overall response rates 2 weeks after the EOT as well as survival on days 14 and 56 were similar in both arms.

In patients with candidemia, the clearance rate of Candida from the bloodstream was similar. Incidence of breakthrough or recurrent infections was slightly higher in the caspofungin group. Success rates in the patients transitioned from IV to oral therapy was $82.6 \%$ in the isavuconazole group and $77.5 \%$ in the caspofungin group. This finding supports the use of isavuconazole as a step-down therapy for candidiasis [46].

\section{Resistance to Isavuconazole}

Similar mechanisms that lead to fluconazole resistance in Candida species also confer resistance to isavuconazole [47]. Emergence of azole-resistant strains can occur after repeated exposure to the drugs within the azole class [48]. The mechanisms for azole resistance include overexpression of efflux pumps through the ATP binding cassette (ABC) transporter overexpression, mutations in the gene coding the target enzyme (ERG11) leading to reduction in the binding of azoles, mutations in the ERG3 gene resulting in the inability of azoles to disrupt the cell membrane [49]. Multiple mechanisms of resistance can be present in a single Candida strain and can lead to cross-resistance among the triazoles [47]. Sanglard et al. found that ABC transporters, specifically, CDr1 and CgCDR1, had the greatest effect on isavuconazole MICs when present in C. albicans. In contrast to fluconazole and voriconazole, isavuconazole MICs were less affected by major facilitator superfamily (MFS) transporters MDR1 or FLU1. Clinical failure during treatment with an azole should prompt the initiation of an alternative antifungal agent [48]. The well-described mechanism of azole resistance in Aspergillus species is alterations in the Cyp51A gene leading to changes in the enzyme targeted by azoles. Other potential mechanisms including efflux pumps and mutations in the promoter region of Cyp51A are less well-described [48]. The in vitro data support cross-resistance to isavuconazole in Aspergillus isolates with Cyp51A mutations conferring resistance to other azoles, such as voriconazole [50]. Mucorales species exhibit varying degrees of sensitivity to isavuconazole, and species identification and MIC testing are recommended prior to initiating therapy with this agent.

\section{Summary}

Isavuconazole has many advantages over other anti-fungal medications, including both IV and oral formulation, broad spectrum activity, predictable pharmacokinetics, and reduced adverse effects when compared to other triazoles. Isavuconazole is an excellent alternative to voriconazole for IA in 
patients with hematologic malignancies with significant concerns for drug interactions and toxicities. It is also a reasonable consideration for a step-down and salvage therapy for mucormycosis in patients with refractory disease and those unable to tolerate posaconazole. Although it was not found to be non-inferior to caspofungin for IC, it is an option for an oral step-down therapy when fluconazole cannot be used. Isavuconazole's role in anti-mold prophylaxis in high-risk patients is less well-defined. Further studies are needed in this area.

Funding: This research received no external funding.

Conflicts of Interest: L.O. received speaking, consulting, and/or research funds from Astellas, Pfizer, Cidara, Amplyx, Scynexis, Mayne, F2G, Viracor, and Gilead. M.E. has no reported conflicts.

\section{References}

1. Brown, G.D.; Denning, D.W.; Gow, N.A.; Levitz, S.M.; Netea, M.G.; White, T.C. Hidden killers: Human fungal infections. Sci. Transl. Med. 2012, 4, 165rv13. [CrossRef] [PubMed]

2. Bongomin, F.; Gago, S.; Oladele, R.O.; Denning, D.W. Global and Multi-National Prevalence of Fungal Diseases-Estimate Precision. J. Fungi 2017, 3, 57. [CrossRef] [PubMed]

3. Gallagher, J.C.; Dodds Ashley, E.S.; Drew, R.H.; Perfect, J.R. Antifungal pharmacotherapy for invasive mould infections. Expert Opin. Pharmacother. 2003, 4, 147-164. [CrossRef] [PubMed]

4. Livermore, J.; Hope, W. Evaluation of the pharmacokinetics and clinical utility of isavuconazole for treatment of invasive fungal infections. Expert Opin. Drug Metab. Toxicol. 2012, 8, 759-765. [CrossRef] [PubMed]

5. Jenks, J.D.; Salzer, H.J.; Prattes, J.; Krause, R.; Buchheidt, D.; Hoenigl, M. Spotlight on isavuconazole in the treatment of invasive aspergillosis and mucormycosis: Design, development, and place in therapy. Drug Des. Dev. Ther. 2018, 12, 1033-1044. [CrossRef]

6. Schmitt-Hoffmann, A.; Desai, A.; Kowalski, D.; Pearlman, H.; Yamazaki, T.; Townsend, R. Isavuconazole absorption following oral administration in healthy subjects is comparable to intravenous dosing, and is not affected by food, or drugs that alter stomach pH. Int. J. Clin. Pharmacol. Ther. 2016, 54, 572-580. [CrossRef]

7. Kovanda, L.L.; Marty, F.M.; Maertens, J.; Desai, A.V.; Lademacher, C.; Engelhardt, M.; Lu, Q.; Hope, W.W. Impact of Mucositis on Absorption and Systemic Drug Exposure of Isavuconazole. Antimicrob. Agents Chemother. 2017, 61, e00101-17. [CrossRef]

8. Schmitt-Hoffmann, A.; Roos, B.; Heep, M.; Schleimer, M.; Weidekamm, E.; Brown, T.; Roehrle, M.; Beglinger, C. Single-ascending-dose pharmacokinetics and safety of the novel broad-spectrum antifungal triazole BAL4815 after intravenous infusions (50, 100, and 200 milligrams) and oral administrations (100, 200, and 400 milligrams) of its prodrug, BAL8557, in healthy volunteers. Antimicrob. Agents Chemother. 2006, 50, 279-285. [CrossRef]

9. Schmitt-Hoffmann, A.; Roos, B.; Maares, J.; Heep, M.; Spickerman, J.; Weidekamm, E.; Brown, T.; Roehrle, M. Multiple-dose pharmacokinetics and safety of the new antifungal triazole BAL4815 after intravenous infusion and oral administration of its prodrug, BAL8557, in healthy volunteers. Antimicrob. Agents Chemother. 2006, 50, 286-293. [CrossRef]

10. Everson, N.; Smith, J.; Garner, D. Successful treatment of contaminated epidural steroid associated fungal menigitis with isauvconazole. In Proceedings of the European Congress of Clinical Microbiology and Infectious Disease (ECCMID), Copenhagen, Denmark, 25-28 April 2015. Abstract P0231.

11. Schmitt-Hoffmann, A.H.; Kato, K.; Townsend, R.; Potchoiba, M.J.; Hope, W.W.; Andes, D.; Spickermann, J.; Schneidkraut, M.J. Tissue Distribution and Elimination of Isavuconazole following Single and Repeat Oral-Dose Administration of Isavuconazonium Sulfate to Rats. Antimicrob. Agents Chemother. 2017, 61, e01292-17. [CrossRef]

12. Kovanda, L.L.; Desai, A.V.; Lu, Q.; Townsend, R.W.; Akhtar, S.; Bonate, P.; Hope, W.W. Isavuconazole Population Pharmacokinetic Analysis Using Nonparametric Estimation in Patients with Invasive Fungal Disease (Results from the VITAL Study). Antimicrob. Agents Chemother. 2016, 60, 4568-4576. [CrossRef] [PubMed]

13. Kaindl, T.; Andes, D.; Engelhardt, M.; Saulay, M.; Larger, P.; Groll, A.H. Variability and exposure-response relationships of isavuconazole plasma concentrations in the Phase 3 SECURE trial of patients with invasive mould diseases. J. Antimicrob. Chemother. 2019, 74, 761-767. [CrossRef] [PubMed] 
14. Townsend, R.W.; Akhtar, S.; Alcorn, H.; Berg, J.K.; Kowalski, D.L.; Mujais, S.; Desai, A.V. Phase I trial to investigate the effect of renal impairment on isavuconazole pharmacokinetics. Eur. J. Clin. Pharmacol. 2017, 73, 669-678. [CrossRef] [PubMed]

15. Zurl, C.; Waller, M.; Schwameis, F.; Muhr, T.; Bauer, N.; Zollner-Schwetz, I.; Valentin, T.; Meinitzer, A.; Ullrich, E.; Wunsch, S.; et al. Isavuconazole Treatment in a Mixed Patient Cohort with Invasive Fungal Infections: Outcome, Tolerability and Clinical Implications of Isavuconazole Plasma Concentrations. J. Fungi 2020, 6, 90. [CrossRef] [PubMed]

16. Zhao, Y.; Seelhammer, T.G.; Barreto, E.F.; Wilson, J.W. Altered Pharmacokinetics and Dosing of Liposomal Amphotericin B and Isavuconazole during Extracorporeal Membrane Oxygenation. Pharmacotherapy 2020, 40, 89-95. [CrossRef] [PubMed]

17. Andes, D.; Kovanda, L.; Desai, A.; Kitt, T.; Zhao, M.; Walsh, T.J. Isavuconazole Concentration in Real-World Practice: Consistency with Results from Clinical Trials. Antimicrob. Agents Chemother. 2018, 62, e00585-18. [CrossRef]

18. Patterson, T.F.; Thompson, G.R., 3rd; Denning, D.W.; Fishman, J.A.; Hadley, S.; Herbrecht, R.; Kontoyiannis, D.P.; Marr, K.A.; Morrison, V.A.; Nguyen, M.H.; et al. Executive Summary: Practice Guidelines for the Diagnosis and Management of Aspergillosis: 2016 Update by the Infectious Diseases Society of America. Clin. Infect. Dis. 2016, 63, 433-442. [CrossRef]

19. Tissot, F.; Agrawal, S.; Pagano, L.; Petrikkos, G.; Groll, A.H.; Skiada, A.; Lass-Flörl, C.; Calandra, T.; Viscoli, C.; Herbrecht, R. ECIL-6 guidelines for the treatment of invasive candidiasis, aspergillosis and mucormycosis in leukemia and hematopoietic stem cell transplant patients. Haematologica 2017, 102, 433-444. [CrossRef]

20. Guinea, J.; Peláez, T.; Recio, S.; Torres-Narbona, M.; Bouza, E. In vitro antifungal activities of isavuconazole (BAL4815), voriconazole, and fluconazole against 1007 isolates of zygomycete, Candida, Aspergillus, Fusarium, and Scedosporium species. Antimicrob. Agents Chemother. 2008, 52, 1396-1400, Erratum in 2008, 52, 4211. [CrossRef]

21. Astvad, K.M.T.; Hare, R.K.; Arendrup, M.C. Evaluation of the in vitro activity of isavuconazole and comparator voriconazole against 2635 contemporary clinical Candida and Aspergillus isolates. Clin. Microbiol. Infect. 2017, 23, 882-887. [CrossRef]

22. Seifert, H.; Aurbach, U.; Stefanik, D.; Cornely, O. In vitro activities of isavuconazole and other antifungal agents against Candida bloodstream isolates. Antimicrob. Agents Chemother. 2007, 51, 1818-1821. [CrossRef] [PubMed]

23. Espinel-Ingroff, A.; Chowdhary, A.; Gonzalez, G.M.; Guinea, J.; Hagen, F.; Meis, J.F.; Thompson, G.R., 3rd; Turnidge, J. Multicenter study of isavuconazole MIC distributions and epidemiological cutoff values for the Cryptococcus neoformans-Cryptococcus gattii species complex using the CLSI M27-A3 broth microdilution method. Antimicrob. Agents Chemother. 2015, 59, 666-668. [CrossRef] [PubMed]

24. Osei Sekyere, J. Candida auris: A systematic review and meta-analysis of current updates on an emerging multidrug-resistant pathogen. Microbiologyopen 2018, 7, e00578, Erratum in 2019, 8, e00901. [CrossRef] [PubMed]

25. Datta, K.; Rhee, P.; Byrnes, E., 3rd; Perlin, D.S.; Staab, J.F.; Marr, K.A. Isavuconazole activity against Aspergillus lentulus, Neosartorya udagawae, and Cryptococcus gattii, emerging fungal pathogens with reduced azole susceptibility. J. Clin. Microbiol. 2013, 51, 3090-3093. [CrossRef] [PubMed]

26. Arendrup, M.C.; Jensen, R.H.; Meletiadis, J. In Vitro Activity of Isavuconazole and Comparators against Clinical Isolates of the Mucorales Order. Antimicrob. Agents Chemother. 2015, 59, 7735-7742. [CrossRef] [PubMed]

27. Maertens, J.A.; Raad, I.I.; Marr, K.A.; Patterson, T.F.; Kontoyiannis, D.P.; Cornely, O.A.; Bow, E.J.; Rahav, G.; Neofytos, D.; Aoun, M.; et al. Isavuconazole versus voriconazole for primary treatment of invasive mould disease caused by Aspergillus and other filamentous fungi (SECURE): A phase 3, randomised-controlled, non-inferiority trial. Lancet 2016, 387, 760-769. [CrossRef]

28. Keirns, J.; Desai, A.; Kowalski, D.; Lademacher, C.; Mujais, S.; Parker, B.; Schneidkraut, M.J.; Townsend, R.; Wojtkowski, T.; Yamazaki, T.; et al. QT Interval Shortening With Isavuconazole: In Vitro and In Vivo Effects on Cardiac Repolarization. Clin. Pharmacol. Ther. 2017, 101, 782-790. [CrossRef]

29. Mellinghoff, S.C.; Bassetti, M.; Dörfel, D.; Hagel, S.; Lehners, N.; Plis, A.; Schalk, E.; Vena, A.; Cornely, O.A. Isavuconazole shortens the QTc interval. Mycoses 2018, 61, 256-260. [CrossRef] 
30. Astellas Pharma US \& Inc. CRESEMBA (isavuconazonium_sulfate) Prescribing Information. Available online: https://www.accessdata.fda.gov/drugsatfda_docs/label/2015/207500Orig1s000lbl.pdf (accessed on 30 September 2020).

31. Kieu, V.; Jhangiani, K.; Dadwal, S.; Nakamura, R.; Pon, D. Effect of isavuconazole on tacrolimus and sirolimus serum concentrations in allogeneic hematopoietic stem cell transplant patients: A drug-drug interaction study. Transpl. Infect. Dis. 2019, 21, e13007. [CrossRef]

32. Kontoyiannis, D.P.; Marr, K.A.; Park, B.J.; Alexander, B.D.; Anaissie, E.J.; Walsh, T.J.; Ito, J.; Andes, D.R.; Baddley, J.W.; Brown, J.M.; et al. Prospective surveillance for invasive fungal infections in hematopoietic stem cell transplant recipients, 2001-2006: Overview of the Transplant-Associated Infection Surveillance Network (TRANSNET) Database. Clin. Infect. Dis. 2010, 50, 1091-1100. [CrossRef] [PubMed]

33. Bongomin, F.; Maguire, N.; Moore, C.B.; Felton, T.; Rautemaa-Richardson, R. Isavuconazole and voriconazole for the treatment of chronic pulmonary aspergillosis: A retrospective comparison of rates of adverse events. Mycoses 2019, 62, 217-222. [CrossRef] [PubMed]

34. Harrington, R.; Lee, E.; Yang, H.; Wei, J.; Messali, A.; Azie, N.; Wu, E.Q.; Spalding, J. Cost-Effectiveness Analysis of Isavuconazole vs. Voriconazole as First-Line Treatment for Invasive Aspergillosis. Adv. Ther. 2017, 34, 207-220. [CrossRef] [PubMed]

35. Floros, L.; Pagliuca, A.; Taie, A.A.; Weidlich, D.; Rita Capparella, M.; Georgallis, M.; Sung, A.H. The cost-effectiveness of isavuconazole compared to the standard of care in the treatment of patients with invasive fungal infection prior to differential pathogen diagnosis in the United Kingdom. J. Med. Econ. 2020, 23, 86-97. [CrossRef] [PubMed]

36. Floros, L.; Kuessner, D.; Posthumus, J.; Bagshaw, E.; Sjölin, J. Cost-effectiveness analysis of isavuconazole versus voriconazole for the treatment of patients with possible invasive aspergillosis in Sweden. BMC Infect. Dis. 2019, 19, 134. [CrossRef] [PubMed]

37. Ullmann, A.J.; Aguado, J.M.; Arikan-Akdagli, S.; Denning, D.W.; Groll, A.H.; Lagrou, K.; Lass-Flörl, C.; Lewis, R.E.; Munoz, P.; Verweij, P.E.; et al. Diagnosis and management of Aspergillus diseases: Executive summary of the 2017 ESCMID-ECMM-ERS guideline. Clin. Microbiol. Infect. 2018, 24, e1-e38. [CrossRef]

38. Petrikkos, G.; Skiada, A.; Lortholary, O.; Roilides, E.; Walsh, T.J.; Kontoyiannis, D.P. Epidemiology and clinical manifestations of mucormycosis. Clin. Infect. Dis. 2012, 54 (Suppl. 1), S23-S34. [CrossRef] [PubMed]

39. Marty, F.M.; Ostrosky-Zeichner, L.; Cornely, O.A.; Mullane, K.M.; Perfect, J.R.; Thompson, G.R., 3rd; Alangaden, G.J.; Brown, J.M.; Fredricks, D.N.; Heinz, W.J.; et al. VITAL and FungiScope Mucormycosis Investigators. Isavuconazole treatment for mucormycosis: A single-arm open-label trial and case-control analysis. Lancet Infect Dis. 2016, 16, 828-837. [CrossRef]

40. Perfect, J.R.; Cornely, O.A.; Heep, M.; Ostrosky-Zeichner, L.; Mullane, K.M.; Maher, R.; Croos-Dabrera, R.; Lademacher, C.; Engelhardt, M.; Chen, C.; et al. Isavuconazole treatment for rare fungal diseases and for invasive aspergillosis in patients with renal impairment: Challenges and lessons of the VITAL trial. Mycoses 2018, 61, 420-429. [CrossRef]

41. Ashkenazi-Hoffnung, L.; Bilavsky, E.; Levy, I.; Grisaru, G.; Sadot, E.; Ben-Ami, R.; Novikov, A.; Fischer, S.; Nahum, E.; Scheuerman, O. Isavuconazole As Successful Salvage Therapy for Mucormycosis in Pediatric Patients. Pediatr. Infect. Dis. J. 2020, 39, 718-724. [CrossRef]

42. Ilharco, M.; Pereira, C.M.; Moreira, L.; Proença, A.L.; do Carmo Fevereiro, M.; Lampreia, F.; Oliveira, M.L.; Rola, J. Rhinoorbital mucormycosis in the immunocompetent: Experience with Isavuconazole. IDCases 2019, 18, e00591. [CrossRef]

43. Shafiq, M.; Ali, Z.; Ukani, R.; Brewer, J. Isavuconazole: A Promising Salvage Therapy for Invasive Mucormycosis. Cureus 2018, 10, e2547. [CrossRef] [PubMed]

44. Ricotta, E.E.; Lai, Y.L.; Babiker, A.; Strich, J.R.; Kadri, S.S.; Lionakis, M.S.; Prevots, D.R.; Adjemian, J. Invasive candidiasis species distribution and trends, United States, 2009-2017. J. Infect. Dis. 2020. [CrossRef]

45. Pfaller, M.A.; Diekema, D.J. Epidemiology of invasive candidiasis: A persistent public health problem. Clin. Microbiol. Rev. 2007, 20, 133-163. [CrossRef] [PubMed]

46. Kullberg, B.J.; Viscoli, C.; Pappas, P.G.; Vazquez, J.; Ostrosky-Zeichner, L.; Rotstein, C.; Sobel, J.D.; Herbrecht, R.; Rahav, G.; Jaruratanasirikul, S.; et al. Isavuconazole Versus Caspofungin in the Treatment of Candidemia and Other Invasive Candida Infections: The ACTIVE Trial. Clin. Infect. Dis. 2019, 68, 1981-1989. [CrossRef] [PubMed] 
47. Wilson, D.T.; Dimondi, V.P.; Johnson, S.W.; Jones, T.M.; Drew, R.H. Role of isavuconazole in the treatment of invasive fungal infections. Ther. Clin. Risk Manag. 2016, 12, 1197-1206. [CrossRef]

48. Sanglard, D.; Coste, A.T. Activity of Isavuconazole and Other Azoles against Candida Clinical Isolates and Yeast Model Systems with Known Azole Resistance Mechanisms. Antimicrob. Agents Chemother. 2015, 60, 229-238. [CrossRef]

49. Pfaller, M.A. Antifungal drug resistance: Mechanisms, epidemiology, and consequences for treatment. Am. J. Med. 2012, 125 (Suppl. 1), S3-S13. [CrossRef]

50. Jørgensen, K.M.; Astvad, K.M.T.; Hare, R.K.; Arendrup, M.C. EUCAST Susceptibility Testing of Isavuconazole: MIC Data for Contemporary Clinical Mold and Yeast Isolates. Antimicrob. Agents Chemother. 2019, 63, e00073-19. [CrossRef]

Publisher's Note: MDPI stays neutral with regard to jurisdictional claims in published maps and institutional affiliations.

(C) 2020 by the authors. Licensee MDPI, Basel, Switzerland. This article is an open access article distributed under the terms and conditions of the Creative Commons Attribution (CC BY) license (http://creativecommons.org/licenses/by/4.0/). 